\title{
L'art du désordre toléré. La police des manifestations en Allemagne fédérale
}

Fabien JOBARD

CNRS-Cesdip

Telle que prêchée dans les écoles de formation de nos élites, l'Allemagne se caractérise par une vertu suprême : la maîtrise au centime près de son budget. " Null Komma Null ", tonnait Theo Waigel, le ministre des Finances de Helmut Kohl - zéro centime de dépassement des déficits publics. Ces dernières années toutefois, c'est un autre aspect des politiques publiques allemandes qui semble avoir été découvert, sur un tout autre terrain, celui des politiques de maintien de l'ordre ${ }^{1}$. Les polices allemandes offriraient en effet un contraste singulier avec les forces de l'ordre françaises, celui de la tempérance, de la communication et de la modération; comme la rigueur allemande s'opposerait à la folie dépensière des finances publiques françaises. Cette émergence inattendue d'un "modèle allemand " policier porte le nom de " désescalade ", l'un des termes majeurs de l'évolution des doctrines de maintien de l'ordre en Allemagne, comme nous l'avions évoqué, avec Olivier Fillieule, dans un article publié lors de la mobilisation contre la loi El Khomri sous la présidence Hollande et qui a beaucoup contribué à l'adoption de ce nouveau lexique en France ${ }^{2}$. Pourtant, le maintien de l'ordre en Allemagne offre parfois un visage moins pacifique, comme l'ont montré les trois jours d'affrontements à Hambourg, ville-Land alors dirigé par Olaf Scholz (actuel successeur de Theo Waigel), lors du Sommet du G20 début juillet 2017. Les cadres policiers français actuels ne s'y trompent pas, qui consultent aujourd'hui plus volontiers leurs homologues de Hambourg que ceux de Berlin, adeptes proclamés de la Deeskalationsstrategie ${ }^{3}$. Qu'en est-il alors véritablement du "modèle allemand de maintien de l'ordre " ? C'est à cette question toute simple que s'emploie de répondre l'article que l'on va lire.

\section{Un droit et une protection constitutionnels}

Vu de France, l'une des singularités majeures du maintien de l'ordre en Allemagne consiste dans le caractère constitutionnel de la manifestation. La Loi fondamentale allemande dispose en effet dans son article 8 que "tous les Allemands ont le droit de se réunir pacifiquement et sans armes, sans autorisation ni déclaration ". Le second paragraphe de ce bref article précise tout de même que la loi peut venir préciser l'exercice de ce droit lorsqu'il s'exerce sur la voie publique (ou, pour citer

\footnotetext{
${ }^{1}$ Frédéric Lebaron, « Maintien de l’ordre », éditorial de Savoir/Agir $n^{\circ} 36,2016$.

${ }^{2}$ Cet article devint en quelques mois l'article le plus lu du site La Vie des Idées (http://www.laviedesidees.fr/Unsplendide-isolement.html) et son adaptation pour le journal Le Monde (édition des 8 et 9 mai 2016) circula beaucoup parmi les cadres de la Préfecture de police. La journaliste Julia Pascual rapportait que le préfet de police Cadot « souhaitait s'inspirer de ce qui se fait en Allemagne à travers le concept de désescalade » et que son successeur M. Delpuech (remplacé en mars 2019 par M. Lallement) faisait de ce concept son principe d'action. Les deux articles évoqués sont " Loi travail : questions autour du maintien de l'ordre ", 23 septembre 2016 et "Maintien de l'ordre : la stratégie de la désescalade », 25 mai 2018, tous deux publiés in Le Monde.

${ }^{3}$ Entretiens avec des policiers de directions centrales, 2019 et 2020. Ces voyages et échanges internationaux de policiers ne sont pas sans rappeler le " tourisme sécuritaire " vers le New York du maire républicain Giuliani et Bratton (son chef de police) des promoteurs de lignes dures face à la délinquance (Jacques de Maillard et Tanguy Le Goff, "La tolérance zéro en France. Succès d'un slogan, illusion d'un transfert », Revue française de science politique, 4, 2009). Le fédéralisme allemand permet toutefois de ventiler les destinations selon les préférences des visiteurs en termes de stratégies policières.
} 
l'expression originale : "à ciel ouvert »). C'est ce qu'a fait la loi de 1953 sur la liberté de réunion (Versammlungsgesetz), avant que, en 2006, la loi de réforme du fédéralisme transfère ces compétences aux Länder. Les conditions d'exercice de la liberté de manifestation sont désormais définies par les Länder qui disposent déjà des compétences de police générale. Lorsqu'une manifestation se déroule dans une ville donnée, le dispositif est conçu et dirigé par la police du Land, qui a sous son commandement les forces de police qui lui sont affectées le temps de l'opération. Coexistent en Allemagne une "Berliner Linie » (une ligne berlinoise), centrée depuis les élections municipales de 2001 sur la doctrine de la désescalade, et une "Hamburger Linie ", misant au contraire sur l'absence de tolérance au désordre et le respect absolu de la loi - ce qui explique pour une large part le déroulement des opérations, très dures (pour un contexte allemand), lors des rassemblements contre le G20 en juillet 2017, lorsque les 7.000 policiers locaux, les 5.500 policiers fédéraux (Bundespolizei) et les 16.700 policiers d'autres Länder (mais aussi des policiers autrichiens) intervenaient sous l'autorité de la police hambourgeoise.

L'autre trait majeur du droit de la manifestation est la décision de principe du Tribunal constitutionnel appelée « décision Brokdorf » (1985), du nom de cette commune désignée pour accueillir une centrale nucléaire et où les autorités locales, en 1981, prirent des arrêtés d'interdiction des manifestations, avant de faire réprimer violemment les rassemblements qui s'y produisirent. Ce sont ces arrêtés et plus généralement le comportement policier qui nourrirent la décision Brokdorf, aujourd'hui pierre angulaire de la doctrine de désescalade allemande. Le Tribunal constitutionnel rappela en effet que la liberté de manifestation était constitutionnelle et précisa même : "Le droit des citoyens de prendre part, au moyen de la liberté de réunion, de manière active à la formation de la volonté et de l'opinion politiques relève des éléments fonctionnels indispensables à toute communauté démocratique ". Ce faisant, le Tribunal souligna le caractère constitutionnellement protégé de toute forme d'action (y compris les blocages ou sit-in, ou les manifestations non déclarées), dès lors que l'intention des organisateurs ne réside pas dans la volonté de détruire. Il exprima à l'égard des pouvoirs publics une obligation d'agir de manière bien intentionnée (" amicales ») à l'endroit des manifestations annoncées ou en cours (versammlungsfreundlich), et posa à l'égard des forces de police une injonction à la coopération avec les manifestants.

Cette décision est la pierre angulaire du maintien de l'ordre. Elle ne constitue cependant pas une lecture naturelle et obligée de la Loi fondamentale, car dans le même temps le gouvernement conservateur de $\mathrm{H}$. Kohl durcissait les conditions de manifestation, en prohibant en 1985 l'armement dit "passif " (protections corporelles) et la dissimulation du visage puis en autorisant, en 1989, les forces de l'ordre à filmer et photographier les manifestants, tout en durcissant les peines prévues pour infractions en manifestation. Le droit a depuis lors peu évolué ${ }^{4}$. Les juridictions ont plus scrupuleusement distingué la manifestation revendicative d'autres formes de réunions collectives, comme les matchs de football ou la Love Parade, définissant strictement la manifestation à portée politique $^{5}$. Dans les années 1990, la juridiction constitutionnelle a explicitement considéré les rassemblements d'extrême-droite comme des manifestations de plein droit, ce qui oblige la police à les protéger des contre-manifestations alors fréquentes ${ }^{6}$. Les pouvoirs publics ont l'obligation

\footnotetext{
${ }^{4}$ Les lois des Länder sur la liberté de réunion sont actuellement en cours d'adoption - voir à ce sujet le dossier de Vorgänge. Zeitschrift für Bürgerrechte und Gesellschaftspolitik, 213, avril 2016.

${ }^{5}$ Fabien Jobard, «Quand droit et politique sont à la fête. La Love et la Fuckparade sous les fourches civilisatrices du droit administratif allemand ", in Pierre Favre et al. (dir.), L'atelier du politiste. Paris, La Découverte/Pacte, 2007.

${ }^{6}$ Sur la police des rassemblements néo-fascistes, voir Bénédicte Laumond, Policy Responses to the Radical Right in France and Germany, Londres, Routledge, 2020. Ces rassemblements agrègent des publics et revendications diverses au fil du temps, posant des difficultés chaque fois spécifiques aux pouvoirs publics et aux juridictions administratives : manifestations de hooligans comme à Cologne en 2014 (voir mon article dans Mediapart : «État de droit, État défait » (15 novembre 2014), manifestations hostiles au déclin du monde occidental (mouvement Pegida, principalement à Dresde) ou récemment les manifestations contre les mesures de police sanitaire (la
} 
d'assurer aux contre-manifestants la possibilité de faire entendre leur colère à leurs adversaires : agir de manière "versammlungsfreundlich" n'est pas seulement une obligation négative (protéger la manifestation), mais aussi une obligation positive (faire en sorte que les destinataires de la protestation soient en mesure d'entendre le message porté par les (contre-)manifestants).

Si nous avons jugé bon de revenir dans le détail sur la jurisprudence constitutionnelle, c'est pour souligner trois dimensions. La première est le caractère sacré qui marque tant le texte (la Loi fondamentale) que la cour (le Tribunal constitutionnel) qui le protège : chose connue, mais qui a des effets tangibles, une décision de principe du Tribunal constitutionnel allemand est dotée d'une effectivité que l'on soupçonne peu en France. Ainsi, la décision Brokdorf a peu à peu amené un changement de la conception des doctrines et surtout de l'enseignement du maintien de l'ordre dans les écoles de police, et les acteurs politiques, jusqu'aux syndicats de police, ne sauraient contester les décisions judiciaires, par exemple de levée d'une interdiction de manifestation ${ }^{7}$. La deuxième remarque est que la cour constitutionnelle pas plus que les juridictions administratives n'hésitent pas à entrer dans le vif de la matière, en prescrivant des modalités générales d'intervention policière ou en détaillant très exactement les parcours autorisés, la distance à laquelle se tiendra la contremanifestation éventuelle, les lieux sur lesquels il convient de ne pas scander de slogans (en raison, par exemple, d'un monument à la mémoire des victimes de la Shoah ou des soldats russes à proximité, etc.). La justice allemande fait entendre sa voix et, pourrait-on dire, ses armes, dans le grondement même de la bataille. Enfin, ces décisions sont opposables : la décision Brokdorf offre un répertoire argumentatif de choix à tout manifestant contestant un dispositif policier qui lui paraît contraire à l'esprit de la Loi fondamentale. II ne s'agit donc pas seulement, en Allemagne, de se tourner vers les juridictions pénales lorsqu'on accuse un policier d'avoir commis des brutalités lors de manifestation, mais de solliciter l'argumentaire de la juridiction constitutionnelle pour faire valoir que telle disposition préventive est contraire à l'injonction à assurer les conditions d'expression politique dans l'espace public.

L'un des effets les plus visibles de la décision Brokdorf est la traduction concrète de la nécessité pour les pouvoirs publics d'agir de manière "freundlich » et d'assurer à tout instant la " coopération " avec les manifestants ${ }^{8}$. Des équipes policières sont ainsi formées en "Kommunikationsteams " et renseignent les manifestants sur l'itinéraire, les formalités, les attentes des autorités policières ou encore les raisons pour lesquelles une banderole vient d'être saisie ou des manifestants interpellés. Les polices allemandes font aussi un usage intense des outils modernes de communication, tels Facebook et surtout Twitter, de manière à informer les manifestants dans le cours de l'action de ce qui se produit et de ce qui est attendu d'eux. La communication s'adresse autant aux manifestants qu'au public. Les autorités de police livrent de manière continue leurs appréciations sur les événements en cours : déplorent la violence, appellent au calme, signalent un agent blessé, une vitre brisée, etc. Ce faisant, la police pèse fortement sur la manifestation elle-même, dont la perception publique est configurée par l'interprétation policière des choses, dotée d'une force de frappe considérable au vu des moyens employés (ce ne sont ainsi pas moins de 300 policiers qui furent affectés lors du G20 à la communication) ${ }^{9}$. La communication/propagande ne détourne pas la police

«Corona-Diktatur »), avec des violences contre des journalistes et des policiers, par exemple à Berlin en août ou Leipzig en novembre 2020.

${ }^{7}$ Sur cet aspect singulier (vu de France), voir mes remarques à propos d'une manifestation d'extrême-droite à Cologne en 2014 ("L'Etat de droit et l'Etat défait », Mediapart, 15 novembre 2014, http://blogs.mediapart.fr/edition/police-co/article/151114/letat-de-droit-et-letat-defait).

${ }^{8}$ Le récent « schéma national du maintien de l'ordre " présenté en septembre 2020 par le ministère français de I'Intérieur est largement inspiré, en cette matière, des pratiques allemandes, y compris au regard de la duplicité de l'outil.

${ }^{9}$ La police pèse ainsi aujourd'hui bien plus sur ce que Patrick Champagne appelait « la manifestation de papier ». Voir à ce propos la table-ronde avec des militants de trois classes d'âge différentes ("Eine kleine Demogeschichte. Protest und Polizei in den letzten vierzig Jahren », Bürgerrechte und Polizei, 100, 2011, p. 60). 
de la communication/pacification de la manifestation. Une étude comparée des formations au maintien de l'ordre révèle que les polices allemandes mettent en pratique dans leurs entraînements la capacité à maintenir le contact et l'échange avec les manifestants, même hostiles ou mal disposés, alors que les mêmes entraînements en France, en Angleterre ou en Irlande visent seulement à aguerrir les effectifs et à les préparer aux situations d'escalade et de violence, au " worst-case scenario ${ }^{10}$.

\section{La tentation de la prévention}

Ces dispositions si favorables au droit de manifester ne doivent cependant pas faire oublier deux dimensions propres à l'expression politique dans l'Allemagne contemporaine. D'abord le souvenir laissé par la République de Weimar et les batailles rangées entre nazis et communistes, parfois entre nazis, communistes et police, sans oublier la répression féroce des révoltes à Berlin ou en Bavière après la Première Guerre mondiale est tenace : pour beaucoup de constituants de l'après-guerre, maîtriser la rue est un impératif démocratique catégorique, qui s'est traduit par une faible tolérance à l'égard de la violence. Ensuite ce souvenir connaît une traduction juridique particulière : I'Allemagne fédérale défend une doctrine de "wehrhafte Demokratie » ou de "démocratie militante ", qui consiste à agir par des politiques publiques préventives fortes, contre toute manifestation de radicalisme, " au risque même de violer certains de ses principes fondamentaux ${ }^{11}$. Cette prévention à l'égard de la dimension éruptive de la rue a diverses implications sur le maintien de l'ordre.

Certaines dispositions préventives comme l'interdiction de la dissimulation du visage et des protections physiques (jambières, plastron, coudières...) ${ }^{12}$ ont été ainsi introduites dans la loi plus tôt qu'en France, à un moment où les divers "Schwarze Blocks" engageaient fréquemment la confrontation physique avec la police (jusque même tuer deux policiers par arme à feu lors des manifestations contre l'extension de l'aéroport de Francfort en 1987). La qualification des protections corporelles en "armes passives" permettait alors d'assurer le caractère constitutionnel de leur incrimination pénale, puisque l'art. 8 de la Loi fondamentale précisait bien que la manifestation s'entend « sans armes».

La prévention de la violence ne s'arrête cependant pas à la fouille des sacs ou l'interdiction de la dissimulation du visage. Dès lors que les autorités établissent un " pronostic " argumenté sur les risques posés par une manifestations à venir (et ce pronostic est bien plus précisément examiné, en cas de contentieux, par les juridictions) ${ }^{13}$, les policiers peuvent se rendre au domicile de personnes précises, désignées comme " dangereuses ", et engager des conversations informelles avec elles pour leur rappeler le cadre de la loi et leur indiquer que les autorités... les ont à l'œil, dans un but à peine masqué de les dissuader de se rendre à la manifestation. Elles sont toutefois très peu nombreuses, au

\footnotetext{
10 Andrea Kretschmann, "Les villes artificielles comme espaces de formation de l'ordre ", Carnets de Géographes, à paraître.

${ }^{11}$ Le concept est développé dès les années 1930 par Karl Löwenstein, de qui Bénédicte Laumond tire cette citation in "La régulation du radicalisme de droite. Une comparaison franco-allemande ", Raisons politiques, 78, 2020, p. 113.

${ }^{12}$ Le gaz étant peu utilisé par les polices allemandes, les lunettes de piscine n'étaient alors pas les premiers outils visés. En revanche, il n'était pas rare de voir dans les années 1970 et surtout 1980 les militants se préparer à l'affrontement avec les policiers (des photographies fameuses dans les rues de Francfort du futur ministre des Affaires Etrangères Joschka Fischer en attestent), en se bardant d'équipements défensifs.

${ }^{13}$ Des « notes blanches » sur tel ou tel organisateur ou de simples appréciations non étayées sur le caractère dangereux de telle organisation sont beaucoup plus aisément qu'en France susceptibles d'amener la justice administrative à casser telle ou telle décision prise en amont de la manifestation (sur la France, par contraste, voir Pauline Laborde, "Liberté de manifestation sous état d'urgence ", in Stéphanie Hennette-Vauchez (dir.), Ce qui reste(ra) toujours de l'urgence, Paris, Institut universitaire Varenne, 2018, p. 199.
} 
plus une demi-dizaine par Land en prévision de gros événements ; parfois aucune ${ }^{14}$. Dans de plus rares cas encore, des arrêtés d'interdiction de paraître (instrument policier assez courant dans la gestion des lieux de deal ou de rassemblements de jeunes) sur les lieux voire dans la ville de la manifestation sont pris contre des personnes précises. Ces mesures de police visent des personnes connues dans les fichiers des services de renseignement ou des services de police ${ }^{15}$ comme des " fauteurs de trouble " ou "favorables à la violence, issues du spectre d'extrême-gauche » à propos desquelles les services estiment qu'existe un risque sérieux qu'elles se livrent à des infractions.

Parfois, mais cette fois dans un temps très voisin de l'action, des mesures de rétention policière ("Ingewahrsamnahe ") sont prises contre des personnes souhaitant se rendre à la manifestation, de même que des interdictions pour des étrangers, même européens, de se rendre dans le pays (62 avant le G20 de Hambourg). Ces mesures se sont surtout développées dans le sillage des mouvements dits "Alter » et plus généralement des sommets internationaux, mais elles furent déjà employées sur des manifestations sans dimension internationale comme les "journées du chaos " de 1996, grands rassemblements punk organisés à Hanovre (300 personnes privées de liberté), ou les rassemblements antifascistes de Saalfeld en 1997 (300 personnes) ou de Lübeck en 1998 (400 personnes) ${ }^{16}$. Compte tenu de la vigilance du juge sur ces mesures, elles sont désormais utilisées (surtout vu de France) avec une grande parcimonie. Ainsi lors du G20 de Hambourg, qui réunissait des dizaines de milliers de contestataires venus de toute l'Europe, seules 449 personnes furent amenées dans le lieu de rétention spécialement aménagé (une ancienne halle de marché), dont les trois quarts furent relâchées sur décision judiciaire. Les polices allemandes disposent ainsi d'un instrument qui ne serait concevable, en France, que dans le cadre de lois d'exception, comme la loi sur l'état d'urgence. Mais il est assorti en Allemagne d'un contrôle du juge beaucoup plus sérieux ${ }^{17}$. Des interpellations de plusieurs milliers de personnes pour simple retenue au poste (ce que l'on a appelé des "gardes à vue préventives", récemment encouragées par le procureur de Paris Rémy Heitz) constitueraient en Allemagne un détournement de procédure difficilement imaginable. Quant au traitement des personnes appréhendées, la déclaration d'un haut responsable policier hambourgeois, Jan Hieber, chef de la très active cellule d'enquête sur les délits commis pendant le $\mathrm{G}_{20}{ }^{18}$, donne une idée de l'écart qui sépare

\footnotetext{
${ }^{14}$ Compte tenu du fait que l'État fédéral n'a pas de compétence en matière de prévention, connaître ces mesures implique de les collecter Land par Land. Les parlementaires Grünen ou Die Linke ont généralement, tant au Bundestag qu'aux parlements des Länder posé les questions nécessaires à la connaissance des pratiques préventives (merci à l'Institut für Protest- und Bewegungsforschung pour leur aide sur ces questions). Les 28 pages de réponse gouvernementale à la question des députés Verts du Bundestag indiquent à elles seules (et à toutes fins utiles) en quoi consiste un contrôle parlementaire des actes de police et de renseignement (BT, Drucksache 18/13535).

${ }^{15}$ Il est important de noter la séparation, en Allemagne, entre services de police et services de renseignement ; même si l'attention constante à l'égard de la violence politique permet de disposer, dans la statistique de police, "d'infractions à motivation politique » (PMK) et de services de "protections de l'État » dans les polices des Länder. Sur toutes ces questions, voir les travaux de B. Laumond déjà cités.

${ }^{16}$ Andrej Wroblewski, Stephen Rehmke, «Sicherheit durch Polizeigewahrsam? Anwendung und Recht des 'Unterbindungsgewahrsams'”, Bürgerrechte und Polizei, 63, 1999.

${ }^{17}$ Ainsi durant le G20, les magistrats du siège ont été appelés à travailler en trois-huit, afin de permettre une appréciation sans délai par le juge du bien-fondé de la rétention. En France, la garde à vue avant présentation (le lendemain matin, par exemple) au procureur permet sans autre forme de contrôle l'éloignement du manifestant hors du lieu de rassemblement. Sur ces questions, voir Philipp Knopp, Peter Ulrich, Michael Plöse, "Hamburger Gitter. Entscheidungsräume über Freiheit und Gewahrsam », 2018 (https://g20.protestinstitut.eu/verhaftungen/).

${ }^{18}$ Cette commission (" SoKo Schwarzer Block »), au départ forte de près de 200 OPJ (un chiffre considérable), fut active durant 15 mois, mena 3.400 enquêtes et identifia 850 suspects, parmi lesquels 73 furent identifiés sur dénonciation (des photographies étaient diffusées par la police assorties du numéro vert de la SoKo, campagne à laquelle le quotidien Bild et son service bild.de ont prêté un concours certain) et quelques uns sur reconnaissance faciale numérique. Des mandats d'arrêts furent également émis dans d'autres pays européens.
} 
les pratiques en Allemagne et en France : «Deux tiers des fouilles (pratiquées dans la halle) ont été des fouilles à nu. C'est difficilement explicable et dans un certain nombre de cas injustifiable ».

Une autre conséquence se traduit dans le cours même de la manifestation: à mesure que les manifestants se voyaient interdits d'équipements défensifs, les policiers chargés du maintien de l'ordre gagnaient en équipements individuels, aujourd'hui comme en France de l'ordre de $15 \mathrm{kgs}$ par agent. Mais là où les pouvoirs publics français ont, depuis la fin des années 1990, réduit les effectifs affectés dans les unités spécialisées en maintien de l'ordre ${ }^{19}$, les pouvoirs publics allemands ont conservé la capacité de déployer un volume de forces suffisant pour encadrer au plus près les manifestants à propos desquels existe une suspicion de propension à la violence ${ }^{20}$. C'est ainsi que s'est développée, dès la fin des années 1980 (à l'occasion par exemple de la libération d'anciens détenus de la Fraction Armée Rouge), la tactique visant à faire défiler les manifestants entre des rangées de policiers en tenue de maintien de l'ordre (selon les cas une à deux, très rarement trois rangées), afin d'assurer une intervention la plus précoce possible. Cette technique, essayée ces derniers temps à Paris $^{21}$, est quasi-systématique pour les manifestations d'extrême-gauche : dans ce cas, les groupes antifascistes manifestent mais derrière de telles rangées de policiers que le sens même de la manifestation (« action de rendre visible ») disparaît. L'impératif de protéger les manifestants peut également conduire les policiers à éloigner la contre-manifestation de la manifestation contre laquelle elle proteste : par cette action de prévention de la violence, la tactique policière contrevient là encore à la lettre de la jurisprudence constitutionnelle ; les tribunaux peinent cependant en la matière à se saisir des faits et la jurisprudence est rare ${ }^{22}$.

Il existe en revanche une jurisprudence établie contre les nasses (Einkesselung), employées pour la première fois à Hambourg en 1986 contre environ 800 manifestants qui furent retenus sur place par la police (la nasse est d'ailleurs parfois nommée la " Hamburger Kessel »). Cette technique est utilisée lors des mouvements de sit-ins, revenus en grâce dans le sillage de Occupy Wall Street en 2011. Ainsi en 2013, 900 manifestants (dont deux députés) furent retenus par la police de Francfort non loin de la BCE durant plusieurs heures, au motif qu'ils étaient équipés d'armes passives (en l'espèce des lunettes de soleil et des parapluies, deux instruments de dissimulation du visage). Le tribunal local, puis le Tribunal constitutionnel estimèrent cette manœuvre policière toutefois recevable, en raison des pronostics établis par la police sur les risques d'exactions et de violences, et des échauffourées sérieuses entre la police et des manifestants quelques instants avant (lesquelles firent, selon les personnels soignants sur place, 275 blessés). Ces opérations de police sont l'occasion de nourrir les fichiers de renseignement sur les " extrémistes de gauche violents ». Si la première nasse, celle de 1986, fut déclarée irrecevable par la justice (et chaque manifestant alors indemnisé de 200 Marks), l'obligation de "protéger " les manifestants de tout risque (décision Brokdorf) ouvre aux nasses un espace de légitimation avéré : si la police démontre que la nasse a permis aux manifestants d'exprimer sans risques leurs revendications en isolant des individus dangereux, alors la justice sera encline à valider l'opération.

\footnotetext{
${ }^{19}$ Olivier Fillieule, Fabien Jobard, Politiques du désordre. La police des manifestations en France, Paris, Seuil, 2020, p. 187-199.

${ }^{20}$ Ce surnombre est facilité par les capacités budgétaires solides des pouvoirs publics allemands et par le fait que les forces de maintien de l'ordre accueillent depuis plusieurs décennies des personnels féminins dans leur rang, en forte proportion.

${ }^{21}$ Effet des échanges récents entre polices allemandes et françaises ? Toujours est-il que l'on a observé ces dispositifs à Paris seulement, la Préfecture de police étant le seul territoire qui peut bénéficier d'une abondance de forces à disposition. La tactique est qualifiée à Paris de "flangardage ».

${ }^{22}$ Un exemple typique sont les manifestations de gauche et droite à Dresde, où les uns et les autres étaient disposés de part et d'autre de l'Elbe, et ainsi certes protégés de toute incursion adverse, à ceci près que les contre-manifestants n'étaient pas en mesure de faire valoir leur message au sens où le Tribunal constitutionnel l'envisage (cf. Peer Stolle, "Die Versprechungen der Versammlungsfreiheit und ihre tatsächlichen Grenzen », Vorgänge, 213, 2016, p. 41).
} 


\section{Le rétablissement de l'ordre}

L'équipement policier a exclu, depuis les années 1970, tout usage de grenades, y compris de grenades lacrymogènes. Lorsque le gaz est employé, il l'est par des policiers individuels au moyen de leur bombe lacrymo. Les lanceurs de balle de défense, incongruité française, sont bien sûr exclus (ils équipent seulement les unités spéciales affectées par exemple aux prises d'otages). Les organisations syndicales policières elles-mêmes y sont hostiles, au point que lorsqu'un responsable du syndicat conservateur DGdP s'exprima en faveur de cette arme dans le Süddeutsche Zeitung (2008), il fut rappelé à l'ordre par son secrétaire fédéral. Parce qu'il disperse les foules sans agresser les corps, le canon à eau qui est privilégié en Allemagne ${ }^{23}$. De leur côté, les équipements défensifs des policiers, qui les ont transformés au fil des années 1990 en tortues Ninja, leur permettent désormais de pénétrer sans crainte dans des cortèges hostiles et procéder à des interpellations, ainsi lors de la manifestation "Welcome to Hell " lors du G20 de Hambourg. Le rapport de forces est inversé par rapport à ce qu'il était encore dans les années 1980 : les manifestants n'ont jamais plus le dessus sur la police. Cependant les policiers sont soumis au port de numéros d'identification, qui exerce ses effets sur la retenue des policiers dans les engagements physiques.

Pour avoir assisté tant aux manifestations du "Premier mai révolutionnaire " à Berlin depuis les années 1990 qu'aux trois jours de rassemblements contre le G20 à Hambourg, je peux affirmer que, par comparaison ${ }^{24}$, I'usage de la force en Allemagne reste mesuré. Comme nous le soulignions en 2016, lorsque la violence se déploie, elle ne se multiplie pas en excès ni physiques (matraquages de personnes à terre...), ni verbaux. Sans qu'il soit besoin ici d'insister, un documentaire tel que celui de David Dufresne ("Un pays qui se tient sage», 2020) donne à voir des images absolument inenvisageables en Allemagne.

\section{Conclusion}

Le tableau ici rapidement esquissé du maintien de l'ordre en Allemagne, fortement empreint de droit constitutionnel et de jurisprudence du fait même de l'importance dans ce pays de l'autorité judiciaire dans la régulation de l'expression politique mais aussi dans le contrôle de la police et de l'Exécutif, ne doit pas faire oublier l'évolution propre des mouvements sociaux d'un côté, de la socialisation policière de l'autre. Du côté des mouvements sociaux en effet, la manifestation s'est imposée dès la fin des années 1970 comme un outil de masse d'expression politique, autour d'organisations majeures pour la stabilité de l'ordre politique comme les églises protestantes, qui avaient par exemple initié de gigantesques manifestations pour la paix (contre l'installation des fusées Pershing) et pris une part active aux mouvements antinucléaires. La décision Brokdorf de la Cour constitutionnelle acte d'une certaine manière la diffusion de la manifestation au-delà des cercles étroits des organisations communistes (parti interdit en 1952), des amicales étudiantes plus ou moins d'extrême-droite ou plus tard du mouvement proche de la RAF ou du Mouvement du 2 Juin. Du côté policier, il est clair que des

\footnotetext{
${ }^{23}$ L'eau peut également être une arme redoutable. A Stuttgart en 2010, un manifestant fut énucléé par un canon à eau réglé sur puissance maximale - l'affaire, très connue, fut portée devant les tribunaux, le Land condamné et la victime indemnisée à hauteur de $120.000 €$.

24 Il est important de souligner : " par comparaison ». Pour la plupart des observateurs allemands à Hambourg au G20 de 2017 (voir par exemple Komitee für Grundrecht und Demokratie, Geschichte der Eskalation eines einwöchigen Protestgeschehens, Cologne, 2018 ou Eskalation. Dynamiken der Gewalt im Kontext der G20Proteste in Hamburg 2017 de I'Institut für Protest- und Bewegungsforschung, Berlin, 2018), ces trois jours de manifestation ont donné lieu à une très forte intensité de violence policière - appréciation tout à fait recevable d'un strict point de vue interne. Lors d'un exposé à l'université de Freiburg (« Polizei und Demokratie, ein Lagebericht aus Frankreich ", 4 février 2020), j'ai dû insister pour certifier que les clichés d'équipes policières en civil, LBD en bandoulière, avaient bien été pris à Paris et non dans quelque lointaine et obscure dictature.
} 
tactiques relèvent d'un autre âge, telles que celle de la police de Brême en 1968 (« leur rentrer dedans, les cogner, les poursuivre ") ou encore la Leberwursttaktik (" tactique du pâté de foie "), défendue à l'occasion de la visite à Berlin du Shah d'Iran par le chef de la police berlinoise en ces termes : " coincer les manifestants de part et d'autre, puis s'enfoncer en plein milieu ${ }^{25}$. Dès le début des années 1970, un certain nombre de chefs de police ont entrepris de rompre avec leurs anciens, directement issus de la Wehrmacht et des organisations paramilitaires hitlériennes. En 1970, on lit dans le magazine professionnel Die Polizei cette déclaration signée par 15 cadres de la police de Basse-Saxe : «Un manifestant n'est pas un fauteur de trouble, mais avant tout un citoyen ${ }^{26}$ et les courants de défense de la police démocratique ne cesseront ensuite de se développer, soutiens des gouvernements SPD ou SPD-Verts désireux eux aussi d'introduire des doctrines de désescalade dans la conduite des opérations policières, comme à Berlin sous le maire Klaus Wowereit à partir de 2001. Cette évolution croisée de la socialisation politique des policiers et de la morphologie des mouvements sociaux font des manifestations en Allemagne un moment respecté d'expression collective, à mille lieux de la peur qu'inspire aujourd'hui chez une part croissante de Français l'idée même d'aller battre le pavé.

\footnotetext{
${ }^{25} \mathrm{Au}$ cours de cette fameuse visite, le 2 juin 1967, un étudiant sans armes, en sandale, fut tué d'un coup de revolver tiré par un policier, donnant lieu entre autres conséquences au groupe armé Mouvement du 2 juin. ${ }^{26}$ Michael Kniesel, « Neue Versammlungsgesetz, neues Versammlungsrecht ? », Vorgänge, 213, 2016, p. 22.
} 